\title{
Acquired Tracheoesophageal Fistula through Esophageal Diverticulum in Patient Who Had a Prolonged Tracheostomy Tube
}

\author{
- A Case Report - \\ Jae Hwan Jung, M.D., Ji Sung Kim, M.D., Yong Kyun Kim, M.D.
}

Department of Physical Medicine and Rehabilitation, Kwandong University College of Medicine, Goyang 412-270, Korea

\begin{abstract}
Acquired tracheoesophageal fistula through esophageal diverticulum is infrequent. We report tracheoesophageal fistula through esophageal diverticulum in a 55-year-old male who had a prolonged tracheostomy tube during 6 months, and a NG tube during 18 months. He suffered from recurrent pneumonia. He complained of a cough associated with eating, and production of sputum mixed with food. To help evaluate the aspiration to the lung and the cause of aspiration, he was tested using gastrointestinal scintigraphy (gastric emptying study), a chest CT scan (pre \& post contrast), and esophagoduodenoscopy. The chest CT scan revealed an acquired tracheoesophageal fistula through esophageal diverticulum, and esophagoduodenoscopy revealed a $3 \mathrm{~mm}$ sized fistula that was located $-33 \mathrm{~cm}$ from the upper incisor. We treated the tracheoesophageal fistula by clipping under esophagoduodenoscopy. The symptoms of fever, cough, and aspiration were no long observed after the clipping was completed.
\end{abstract}

Key Words Tracheoesophageal fistula, Diverticulum, Tracheostomy

\section{INTRODUCTION}

Tracheoesophageal fistula can be defined as an abnormal passage between the esophagus and the trachea which can be caused primarily or secondarily. Tracheoesophageal fistula is a very rare disease, and its cause varies according to the age of patients. The disease occurs mostly from natural causes, and afflicts 1 in every

Received November 10, 2009; Accepted June 6, 2010

Corresponding author: Yong Kyun Kim

Department of Physical Medicine and Rehabilitation, Kwandong University College of Medicine, 697-24, Hwajeong-dong, Deogyang-gu, Goyang 412-270, Korea

Tel: 82-31-810-6450, Fax: 82-31-810-5109, E-mail: ykkim@kwandong.ac.kr Copyright @ 2011 by Korean Academy of Rehabilitation Medicine
2,000-4,000 people. ${ }^{1}$ Tracheoesophageal fistula is known to accompany esophageal atresia in infancy and early childhood. The incidence of acquired disease is unknown because it is very rare, but there have been many cases reported in adults. Tracheoesophageal fistula can also be caused by a malignant tumor in the esophagus and the neighboring organs, infectious diseases including tuberculosis, esophagus diverticula, inhalation of foreign substances, or dilatation in areas of stenosis. In cases of non-malignant tracheooesophageal fistula, endotracheal intubation is known as the most common cause. ${ }^{2,3}$ Secondary causes of this disease include, impact and damage on the trachea and the esophagus walls due to the long-term presence of a tracheostomy tube, and a 
few cases have resulted from esophagus diverticula. ${ }^{1}$ Recently, we found tracheoesophageal fistula accompanied by esophagus diverticula in a 55-year-old male patient suffering from recurrent pneumonia with chronic cough, pyrexia, and fitful cough during food intake, by using gastric emptying scintigraphy, chest computed tomography, and esophagoduodenoscopy. The patient's symptoms improved after clipping of the fistula with an endoscope. This study will examine and report the clinical aspects of this case and offer case-related opinions.

\section{CASE REPORT}

A 55-year-old male patient, diagnosed as quadriplegia by intracerebral hemorrhage resulting from rupture of a cerebral aneurysm on the left middle portion of the brain, was hospitalized in the rehabilitation medicine department of our hospital to receive expansive rehabilitation. There was no past history of high blood pressure, diabetes, tuberculosis or hepatitis, but he had a smoking history of 20 years and a clinical history of recurrent pneumonia accompanying pleural effusion. On physical examination, crackling was auscultated at the lower right lung and there was no special diagnosis except dyspnea. On October 30, 2007, 4 days after an operation for intracerebral hemorrhage, tracheotomy was conducted and the patient remained with the tracheostomy tube for 6 months, until May, 2008. When he was hospitalized at my clinic on 12th, June, 2009, a nasogastric tube had been continually inserted for $>1.5$ years and was used for nutrition intake after the intracerebral hemorrhage. A videofluoroscopic swallowing study (VFSS) was conducted and a slight aspiration was observed with solid and semi-solid foods. However, when using a compensation technique by tossing his head toward the paralyzed side, it was confirmed that food entered the esophagus without aspiration. After removing the nasogastric tube and offering sufficient education regarding the compensation technique, oral feeding was started. Since then, when eating using the compensation technique, there were incidents of fitful cough, sputum, fever, and a chest $\mathrm{x}$-ray revealed increased haziness on the left lung, so antibiotic treatment was started under the diagnosis of aspiration pneumonia. However, there was no improvement, so gastric emptying scintigraphy was conducted for detecting other etiologies (Fig. 1). On the gastric emptying scintigraphy, a leak of contrast medium was observed in the middle of the esophagus to the right, and tracheoesophageal fistula was suspected. Therefore, CT was conducted on the chest for an exact diagnosis (Fig. 2). On the image of the chest CT, a cavitary lesion containing $1.8 \mathrm{~cm}$ sized air was observed in the lower part of the right neck, at the level of middle part of the esophagus. Tracheoesophageal fistula was confirmed and upper gastrointestinal endoscopy was conducted to identify the exact size and location (Fig. 3-A). The hole of a tracheoesophageal fistula to the side of the esophagus was in the middle part of the esophagus diverticula, which existed at the $1 / 3$ spot of the middle esophagus, $33 \mathrm{~cm}$ away from eye tooth, and air was observed. It was possible to detect a passage between the esophagus

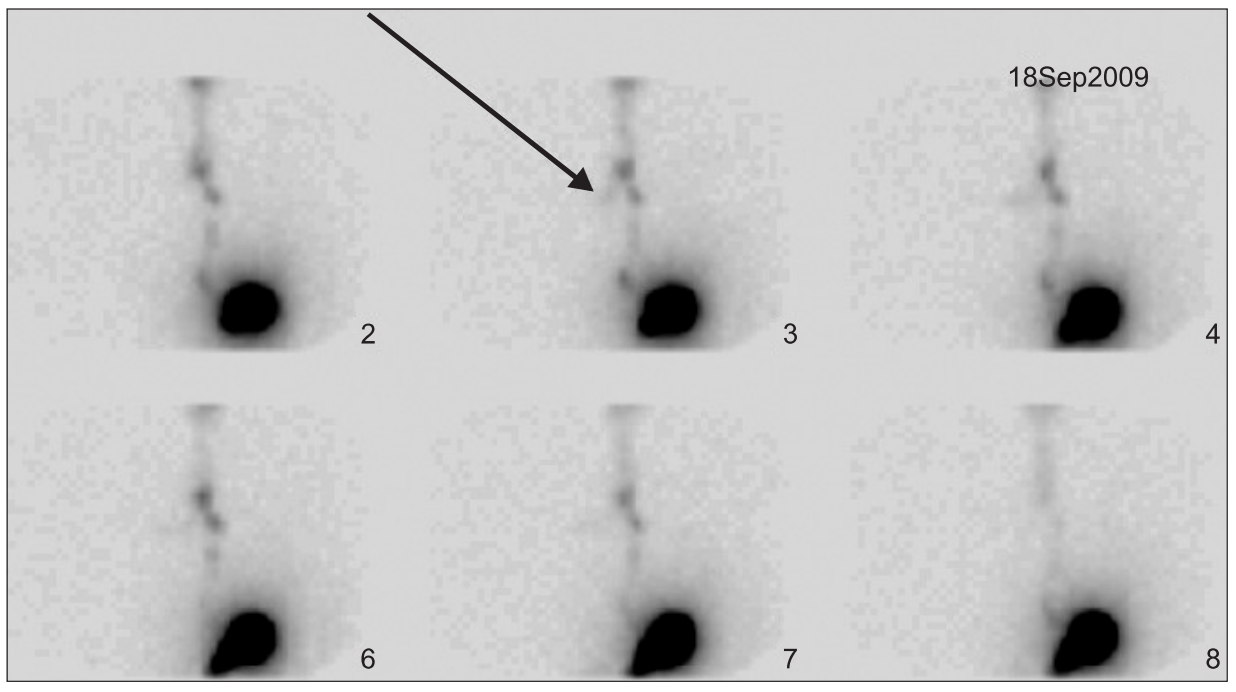

Fig. 1. Tc99m-DTPA was swallowed and serial images were obtained. Gastrointestinal scintigraphy revealed faint tracer activity in right side of mid esophagus (Arrow: faint tracer activity). A tracheoesophageal fistula was suspected in this study. 
and the trachea. The size of the tracheoesophageal fistula was measured as $3 \mathrm{~mm}$ and the leak of contrast medium (Gastrograffin) was observed during the upper gastrointestinal endoscopy. At that time, endoscopic ligation using clips was conducted (4 in total), and the absence of a leak was confirmed using contrast medium (Gastrograffin) (Fig. 3-B). However, in order to eliminate the possibility that the tracheoesophageal fistula was clogged due to inflammation resulting from mucous membrane irritation and edematous changes from endoscopic ligation, an upper GI series was conducted after inflammation and edema subsided on the third day of ligation of the tracheoesophageal fistula (Fig. 4). An upper GI series confirmed there was no leakage of contrast medium and the patient once again started dysphagia meals after a period of continual fasting. Since that time, fever has not been observed, the amount of

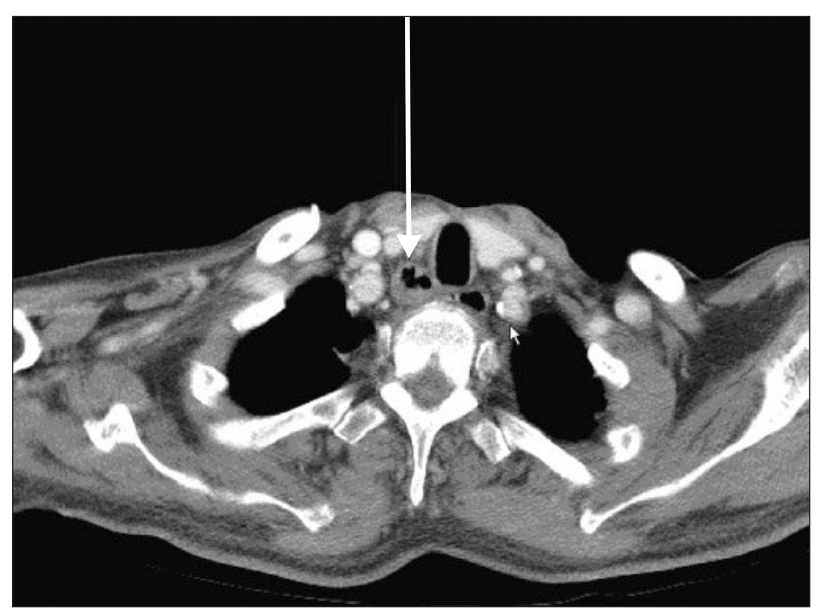

Fig. 2. Transverse chest CT scan revealed the lower neck area mid-esophagus. About $1.8 \mathrm{~cm}$ size cavitary lesion was noted in right lower neck at the level of thoracic inlet (arrow). The esophagus was communicated with trachea. sputum decreased, and there are no clinical features such as aspiration.

\section{DISCUSSION}

According to 44 cases studied by Harley in 1972, tracheoesophageal fistula was observed in -1 patient per $200(0.5 \%)$ who had received a tracheotomy. ${ }^{4}$ Reflux of G-I tract contents may expand a tracheoesophageal fistula by chemical irritation. A nasogastric tube, which is placed to prevent aspiration of G-I tract contents and food,
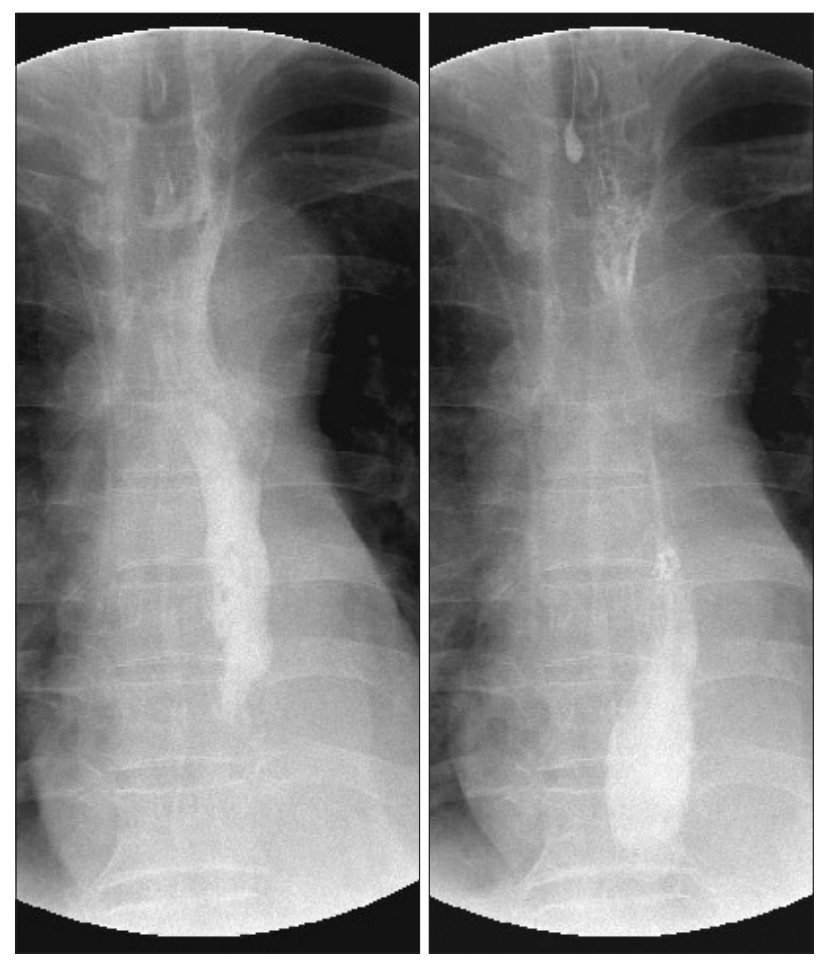

Fig. 4. Endoscopic clipping was done. After 3 days, upper GI series by using gastrograffin revealed that the leakage from mid esophagus was not observed.
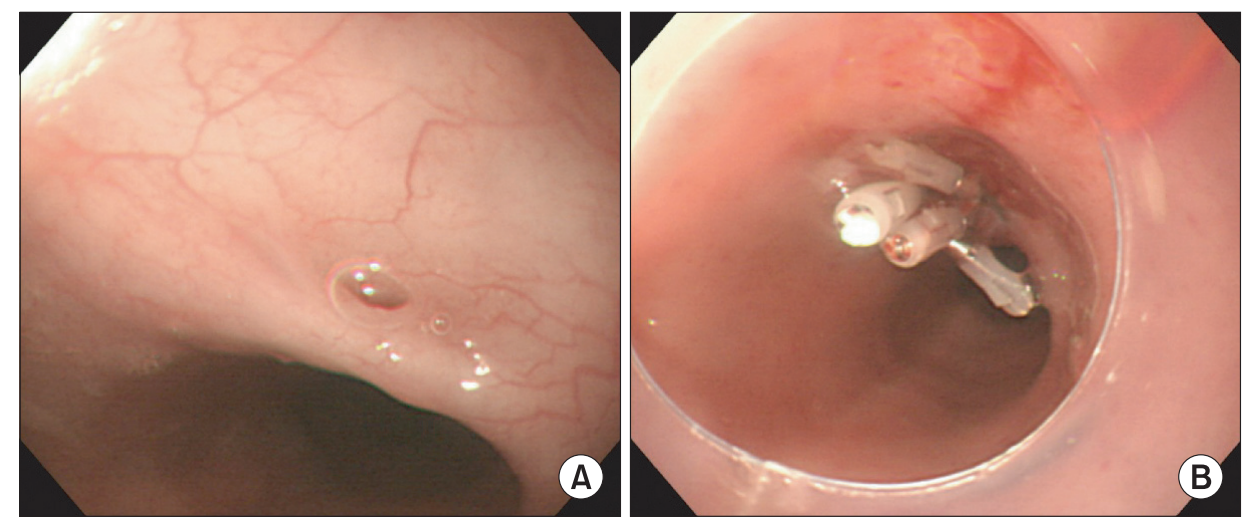

Fig. 3. Esophagoduodenoscopic images: About the $3 \mathrm{~mm}$-sized tracheoesophageal fistula was seen. And also the bubble from the trachea was observed. The fistula was located in the esophagus about 33 $\mathrm{cm}$ from the upper incisor (A). Post endoscopic clipping. Endoscopic clipping was done by using 4 clips (B). 
may increase the incidence of tracheoesophageal fistula because it delivers pressure directly on the partial region of the esophagus. ${ }^{5}$ The risk factors for tracheoesophageal fistula are known to include excessive motion of the endotracheal tube, inflammation, hypotension, diabetes, and long-term use of steroids. Damage of mucous membranes caused by an endotracheal tube can result in destruction of mucous membranes and cartilage, causing tracheomalacia and fibrous stenosis. Later, perforation occurs between the membranous portion of the trachea and the front part of the esophagus, leading to formation of a tracheoesophageal fistula. ${ }^{6}$ In the patient described above, tracheoesophageal fistula occurred while having a nasogastric tube and tracheostomy tube for a long period of time after tracheotomy. The fistula was observed at the height of the trachea and there was severe pain in the middle part of the esophagus diverticula, or the $1 / 3$ spot of the middle esophagus. By inspecting for clinical features and using diagnostic methods, Bertelsen described 3 symptoms of adult tracheoesophageal fistula not accompanying esophageal atresia (H-type). First, airway obstruction or cough while taking meals. Second, recurrent pneumonia or respiratory infection. Third, periodic abdominal distension by air. ${ }^{7}$ In this case, cough and respiratory infection while taking meals occurred most frequently and periodic abdominal distension wasn't observed. It's not easy to diagnose tracheoesophageal fistula using a simple chest x-ray, and diagnosis may require an upper GI series using contrast medium. ${ }^{3}$ However, it's not easy to diagnose this disorder at an early stage, and there are many cases where diagnosis is done after the start of inflammation in the lungs during mechanical ventilation or after death. ${ }^{7}$ The most desirable diagnostic method is to confirm the existence and degree of a fistula through the esophagus or by bronchoscopy. ${ }^{3,8}$

There are various treatments for tracheoesophageal fistula but the basic principle is to solve two problems consisting of respiratory infection and nutrition. In tracheoesophageal fistula, a spontaneous cure is rare and often accompanies tracheostenosis, therefore, surgery is prioritized. However, there are many cases where the condition of the whole body isn't good or pneumonia is accompanied before tracheoesophageal fistula occurs, so it's important to decide the term and method of surgery. ${ }^{9}$ There are 3 types of surgical methods. First, esophageal exclusion to separate air ducts and the esophagus. Second, esophageal stenting to insert a stent into the tracheoesophageal fistula and cut the connection between the trachea and esophagus. Third, direct fistula closure. In case of esophageal exclusion, it makes normal deglutition possible but has the limitation that a patient must have the physical condition to endure surgery under general anesthesia. ${ }^{10}$ In case of esophageal stenting, there are reports that it has fewer side effects and a lower death rate, but the anti-fistular effect is temporary. ${ }^{8}$ In this case, because the condition was complicated by pneumonia, and considering the risks and side-effects of general anesthesia and surgery, direct fistula closure was conducted using clips under esophagoduodenoscopy. Subsequently, no leak of contrast medium was observed from an upper GI series which was conducted 3 days after the procedure to confirm success of clipping, under the condition that inflammation had improved.

The expected complications are tracheostenosis (2.5$22 \%)$, pneumonia, fistula recurrence (7.3-11.4\%), and leak of the esophageal anastomosis (10\%). In this patient's case, after fistula closure using esophagoduodenoscopy, he began oral dieting without complications, and no increase of aspiration or sputum was observed. This report describes a case of tracheoesophageal fistula which was revealed as recurrent pneumonia and accompanied with esophagus diverticula in a patient having a tracheostomy tube for a long period of time. The report also describes the patient's improvement through clipping under esophagoduodenoscopy, rather than through esophagus surgery under general anesthesia, and also provides a literature review.

\section{REFERENCES}

1. Kim KH, Lee JS, Yang HW, Kim JH, Kim GH, Lee MS, Kim WJ. A case of tracheoesophageal fistula through esophageal diverticulm combined with aspiration pneumonia. Korean J Gastroenterol 1997; 30: 677-683

2. Hilgenburg AD, Grillo HC. Acquired nonmalignant tracheoesophageal fistula. J Thorac Cardiovasc Surg 1983; 85: 492-498

3. Jung YH, Baek CW, Park JW, Woo YC, Koo GH. Acquired-tracheoesophageal fistula observed after ventilator care. Korean J Anesthesiol 2004; 46: 122-126

4. Salmon LF. Tracheostomy. Proc R Soc Med 1975; 68: 
347-356

5. Kim SC, Ha KW, Wang JH, Kim SJ, Kim WH, Jeong SH, Lee WS, Han SD, Chon GR. Two cases of postintubation tracheoesophageal fistula in patients with a history of tracheostomy. Korean J Crit Care Med 2009; 24: 87-91

6. Paik HC, Kim DH, Cho HM, Lee DY. Clinical analysis of acquired tracheoesophageal fistula. Korean J Bronchoesophagol 2002; 8: 61-65

7. Bertelson S, Congenital oesophago-tracheal fistulas in the adult. Scand J thorac Cardiovasc Surg 1970; 4: 8082

8. Yeh CM, Chou CM. Early repair of acquired tracheoesophageal fistula. Asian Cardiovascular Thorac Ann 2008; 16: 318-320

9. Jeon SH, Park SW, Jung SW, Lee HR. Postintubation tracheo-esophageal fistula. Korean J Thorac Cardiovasc Surg 1996; 29: 235-238

10. Reed MF, Mathisen DJ. Tracheoesophageal fistula. Chest surg Clni N Am 2003; 13: 271-289 